\title{
Endoscopic management of complicated bacterial orbital cellulitis
}

\section{Abstract}

The aim of this case report is to highlight the management approach to the cases of bacterial orbital cellulitis, a rare entity with serious ophthalmologic and rhinological implications.

In the cases discussed here, the first case is of a 73yrs old male suffering from diabetes mellitus and second case is of $45 \mathrm{yrs}$ HIV positive female. Both patients presented with pain and swelling of eyelids, conjunctival chemosis, proptosis and reduced visual acuity. They underwent nasal endoscopic drainage of the abscess and orbital decompression. First patient also underwent simultaneous repair of CSF Leak using nasal septal cartilage and bone and second patient who had lower eyelid necrotizing fascitis underwent lateral canthotomy followed by tarsorrhaphy. Both the patients improved postoperatively.
Volume 2 Issue 5 - 2015

ID Singh, JR Galagali, KD Joshi, S Mokamati

Department of Otolaryngology Head \& Neck surgery,

Command Hospital (Southern Command), India

Correspondence: Lt Col ID Singh, Department of

Otolaryngology Head \& Neck surgery, Command Hospital

(Southern Command), Pune, India 4l 1040, Tel +91-7767834I37,

Email dridsingh@hotmail.com

Received: February 12, 2015 | Published: May 14, 2015

Keywords: orbital cellulitis, bacterial, immune-compromised, diabetes, HIV, necrotising fasciitis, multidrug resistance, csf leak, sinusitis

\section{Introduction}

Bacterial orbital cellulitis in adults is a rare condition especially eyelid necrotizing fascitis. Recognition of the ocular adnexal changes and characteristic findings on imaging are essential in making this diagnosis. Chandler et al classified the orbital cellulitis in five classespreseptal cellulitis, orbital cellulitis, subperiosteal abscess, intraorbital abscess and cavernous sinus thrombosis. The patient usually presents with fever, proptosis, visual loss and decreased ocular motility. Early treatment is warranted to prevent complete opthalmoplegia with blindness. We describe two peculiar case of orbital cellulitis in an Immunocompromised adult with favourable outcome.

\section{Case description}

\section{Case I}

A 45 yrs old female, known case of HIV, not on antiretroviral therapy, presented with complaints of painful swelling around left eye with severe lower eyelid bacterial necrotizing fascitis which is very rare presentation and gradual loss of vision for one week (Figure 1).

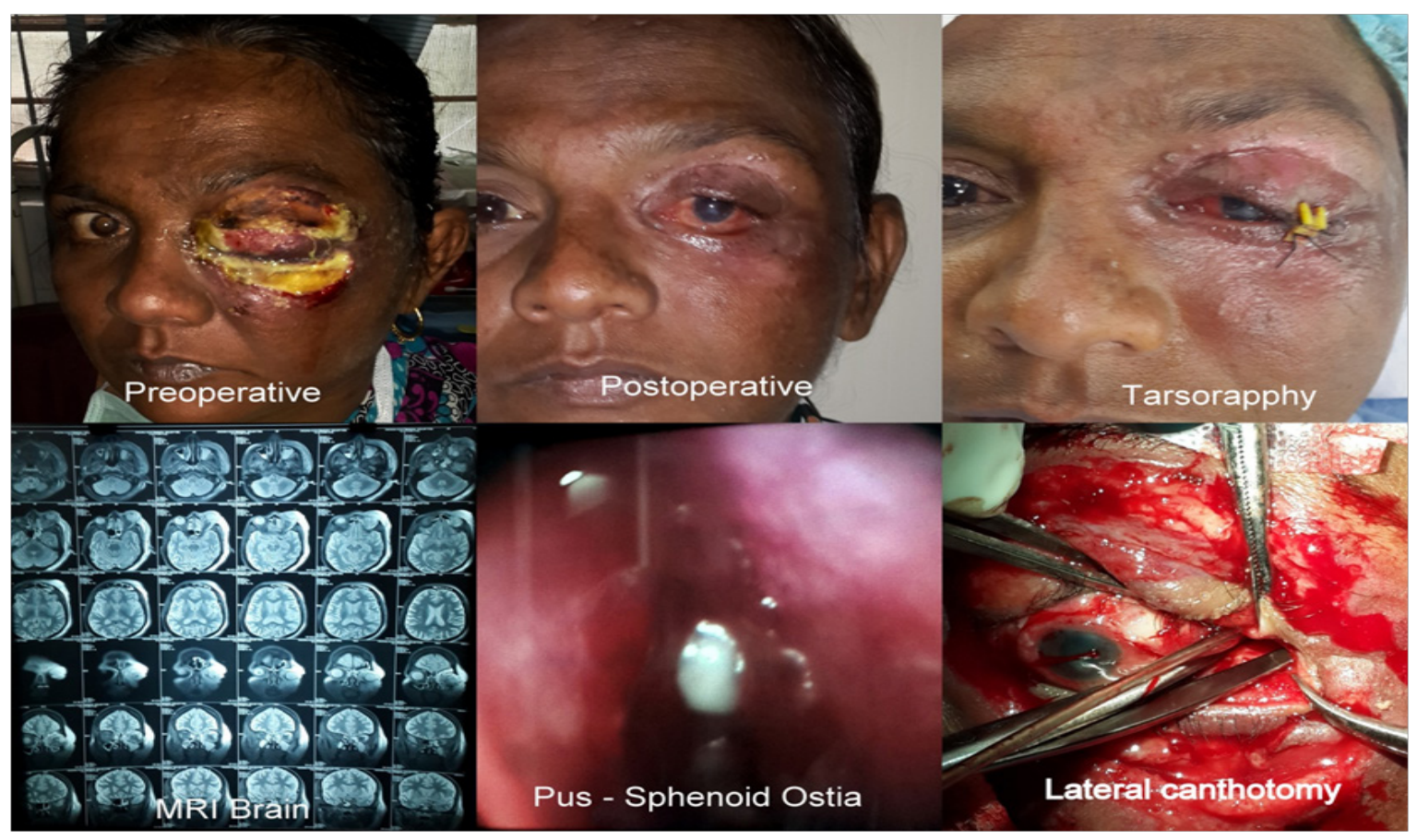

Figure I Preoperative, intraoperative and postoperative pictures of case I. 
Examination revealed exophthalmos, decreased extra ocular movements and only perception of light in with sluggish papillary reflex were present in left eye. Right eye was normal. Frank purulent discharge noted from spheno-ethmoidal recess during nasal endoscopic examination.

Contrast enhanced CT scan was suggestive of left maxillary sinusitis with evidence of subperiosteal collection in left orbit MRI brain revealed proptosis of left eyeball with preseptal and periorbital soft tissue swelling and signal abnormality associated with inflammatory mucosal thickening in left side of paranasal sinuses.

Bacteriological growth from nasal swab showed Pseudomonas aeruginosaresistant to amoxicillin-clavulanic acid, piperacillin -tazobactam,ticaracillin -sulbactum, cefuroxime, ceftriaxone, cefperazone- sulbactum, cefepime, ertapenem, meropenem, amikacin, gentamicin, nalidixic acid, ciprofloxacillin,ticarcillin, nitrofurantoin, colistin, trimethoprim -sulfamethoxazole was detected.Serological investigation were positive for HIV1 and Toxoplasma.

Medical management: ceftazidimeimipenam, clindamycin, metrinidazole

Surgical management: urgent nasal endoscopic sinus surgery with clearance of maxillary, ethmoidal, frontal and sphenoidal sinuses and endoscopic removal of medial orbital wall was done by removing lamina papyracea from superior aspect of maxillary ostia to the superolateral part of the roof of sphenoid sinus, the left orbit entered beyond orbital fat till medial rectus to decompress it adequately into nasal cavity and sphenoid sinus by removing almost entire lamina papyracea and into roof of maxillary sinus by removing inferio-medial wall of the orbit. Sphenoid sinus opened widely up to orbital apex and optic nerve decompressed by incising along its sheath. In addition, lower eyelid wound debridement along with lateral canthotomy was done. Her eyesight improved postoperatively and she underwent staged tarsorrhaphy later on.

\section{Case 2}

A 73 yrs old male having uncontrolled diabetes mellitus presented with complaints of complete loss of vision and painful swelling around right eye for three weeks. On examination the patient had exophthalmos with complete opthalmoplegia with no perception of light in right eye. Mucopus noted from middle meatus and sphenoethmoidal recess during nasal endoscopic examination (Figure 2). Contrast Enhanced CT showed intra-orbital extension of infection in right orbit with thickening of right optic nerve, presumably from adjacent mucosal disease with left parietal subdural collection. There was extensive mucosal involvement of sphenoid, maxillary and ethmoidal sinuses bilaterally. Bacteriological growth from nasal swab showed Staphylococcus aureus.

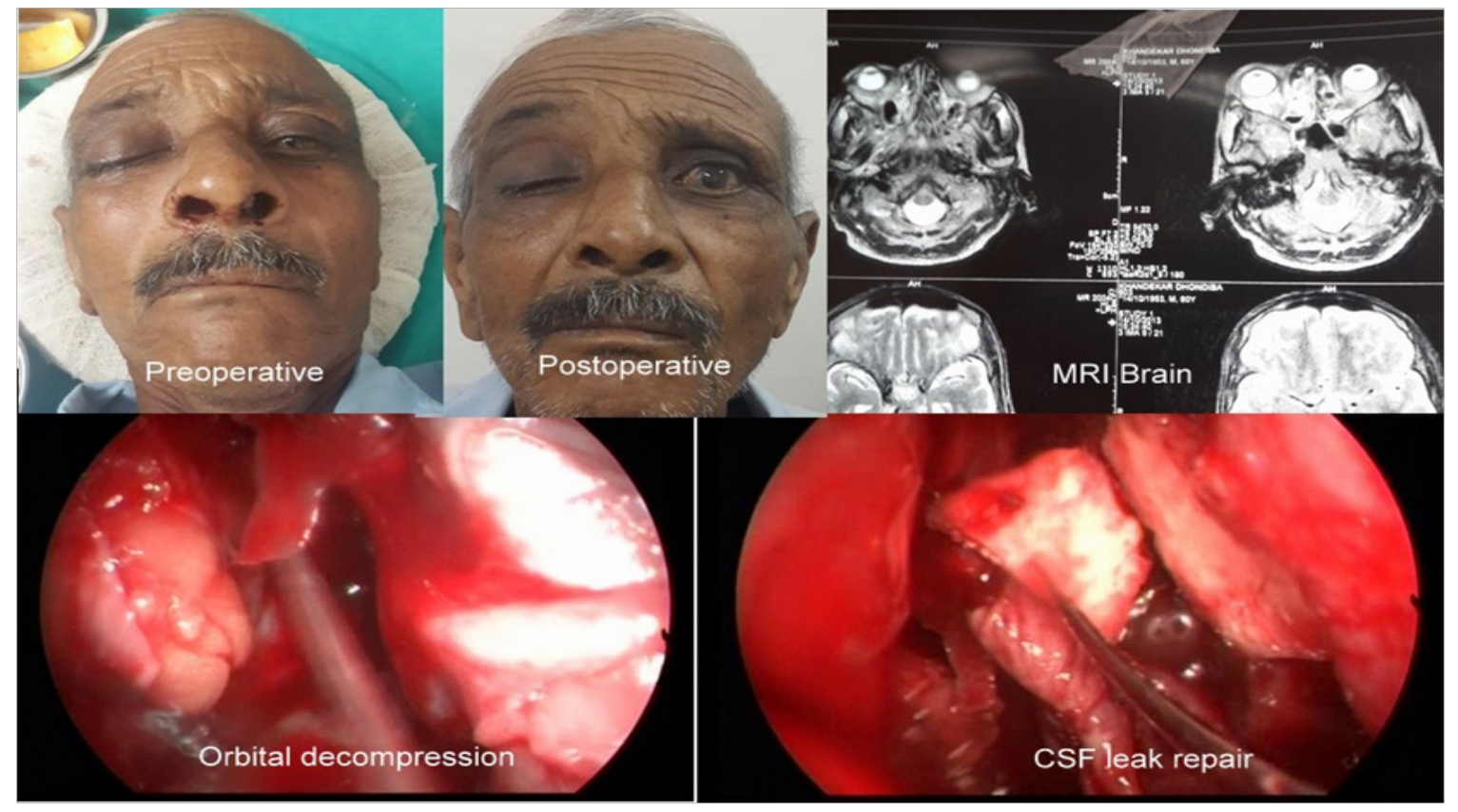

Figure 2 Preoperative, intraoperative and postoperative pictures of case 2.

Medical management: linezolid, clindamycin, cefoperazone, metrinidazole

Surgical management: Urgent endoscopic sinus surgery and clearance of all sinuses and nasal endoscopic medial decompression of the right orbit into nasal cavity and optic nerve decompression by incising along its sheath near orbital apex was done.

Intraoperative dura was unhealthy and granulating just above and lateral to frontal recess roof near the base of anterior cranial fossa. The unhealthy dura with some granulation tissue was removed leading to purposeful iatrogenic CSF leak.

CSF leak repair: Traditionally fascia, muscle, fat graft or mucoperichondriun is used to repair such CSF leaks. In this case, an endoscopic septoplasty was done and the CSF leak was repaired by 'sandwich technique' using two layers of nasal bone and one layer of septal cartilage. Each layer reinforced with Fibrin tissue sealant and finally covered by middle turbinate medial mucosal flap which 
was rotated superiorly. CSF leak was checked by doing valsalva manoeuvre by the ventilating machine and nasal merogel pack given.

Postoperatively patient had favourable recovery with return of flickering movements in his upper eyelid and some perception of light in his operated eye. He had no signs of any CSF leaks and serial nasal endoscopy showed good healing and mucolisation of nasal cavity with no CSF leaks even on straining.

\section{Discussion}

Orbital cellulitis is infectious process with abscess formation in the orbit with breach of periosteum. Clinically it manifests as painful swelling of eyelids, chemosis, decreased ocular motility, diplopia, and proptosis. It can result in blindness, cavernous sinus thrombosis, meningitis and death. ${ }^{1,2}$ The differential diagnosis of orbital cellulitis is given in Table 1.

Predisposing factor in development of orbital cellulitis is sinus pathology followed by trauma, enophthalmosis, orbital implant, dacrocystitis, retained foreign body and dental infection. Paranasal sinus pathology is most commonly implicated in development of orbital cellulitis. Route of spread of infection is local spread or haematogenous spreads. $^{3-5}$ Immunocompromised individuals generally have higher rates of complication and overall poor outcome. ${ }^{6}$ Staphylococcus aureus, Streptococci and Hemophilus influenzae, are the most common microorganism implicated in orbital cellulitis, and older patients the infection is generally by mixture of aerobes and anaerobes. Pseudomonas aeruginosa is a rare cause of orbital cellulitis and present with eyelid necrosis. In immunocompromised patients fungal infections are often complicate the issue. ${ }^{7}$

Various imaging modality include ultrasonography and CT scan can be used. Although a USG is quick and non-invasive but CT scan may be required to assess the evidence of sinusitis and orbital pathology and remains investigation of choice. MRI is indicated for assessing orbital abscess and cavernous sinus thrombosis and if CT scan is contraindicated. ${ }^{3,4,7}$

Medical management includes broad spectrum IV antibiotic initially and then based on culture and sensitivity. Culture from conjunctiva and preseptal tissue might not be representative. However in majority surgical intervention is not required, as it was suggested earlier. Puri et al mentions that timely surgical debridement can decrease the mortality rate to $16-36 \%$ and delay in surgical therapy increases mortality to $73 \%{ }^{8}$ According to Potter et al only $36 \%$ of patient required surgical intervention out of 78 diagnosed cases. ${ }^{9}$ Indication of surgical intervention is non-resolving infection on medical management, demonstration of abscess and presence of complication like visual compromise. .,9,10 $^{4}$

An endoscopic approach is favoured if orbital cellulitis is arising as a complication of sinus disease. The surgical management includes the urgent nasal endoscopic wide medial and inferior orbit decompression with the aim of orbital drainage, optic nerve decompression, lateral canthotomy and in some cases an orbital exenteration, along with adjuvant procedure like endoscopic sinus surgery to remove the underlying cause... The nasal endoscopic approach is safe and quick way of addressing the severe sub-periosteal abscess as well as sinus infections involving the immuno comprised patients with poor general health conditions. The medial decompression is achieved by removal of lamina papyracea, part of superolateral part of sphenoid sinus wall and inferiomedial wall of the orbit so that orbital contents can herniated into nasal cavity, sphenoid sinus and maxillary sinus. The other advantages of nasal endoscopic approach are to do optic nerve decompression and repair of skull base or CSF leaks simultaneously. However in presence of intracranial complications a combined approach is recommended.

\section{Summary}

Orbital cellulitis unlike children is a rare condition in adult. A prompt recognition and treatment is necessary to avoid complication and death. ${ }^{8}$ In present case series one patient recovered fully with no residual vision loss. However one patient had permanent vision loss due to late presentation for treatment. Aggressive medical and surgical management was key in effective treatment of a potentially fatal condition.

\section{Acknowledgments}

None.

\section{Conflicts of interest}

All authors have none to declare. Appropriate permission from the concerned patients have been taken for publication of all the photos and related data.

\section{Funding details}

None.

\section{References}

1. Chandler JR, Langenbrunner DJ, Stevens ER. The pathogenesis of orbital complications in acute sinusitis. Laryngoscope. 1970;80(9):1414-1428.

2. Pandian DG, Babu RK, Chaitra A, et al. Nine years' review on preseptal and orbital cellulitis and emergence of community-acquired methicillin-resistant Staphylococusaureus in a tertiary hospital in India. Indian J Ophthalmol. 2011;59(6):431-435.

3. N Ezhilvathani, Thiagarajan, $\mathrm{T}$ Cherian. An Orbital Cellulitis Demanding Multispeciality Management. The Internet Journal of Ophthalmology and Visual Science. 2009;8(1).

4. Chaha K, Chinda D, Ajike SO. Management of bilateral orbital cellulitis in a 41-year-old man. Niger J Ophthalmol. 2014;22(1):50-55.

5. Liu IT, Kao SC, Wang AG, et al. Preseptal and Orbital Cellulitis: A 10-Year Review of Hospitalized Patients. J Chin Med Assoc. 2006;69(9):415-422.

6. Johnson TE, Casiano RR, Kronish JW, et al. Sino-orbital aspergillosis in acquired immunodeficiency syndrome. Arch Ophthalmol. 1999;117(1):57-64.

7. Lam Choi VB, Yuen HK, Biswas J, et al. Update in Pathological Diagnosis of Orbital Infections and Inflammations. Middle East Afr J Ophthalmol. 2011;18(4):268-276.

8. Puri P, Innes JR. Necrotising fasciitis of orbit; early recognition and treatment a key to success. Eur J Ophthalmol. 2001;11(2):180-182.

9. Potter NJ, Brown CL, McNab AA, et al. Orbital Cellulitis: Medical and Surgical Management. J Clinic Experiment Ophthalmol. 2011;S2:001.

10. Slack R, Sim R, Scott- Browns Otorhinolaryngology head and neck surgery, Seventh edition; Edward Arnold publishers Ltd 2008. 